\title{
A PROBLEM IN LIE RINGS
}

\author{
by JAMES WIEGOLD
}

(Received 12 April, 1979)

An important step in the proof of Kostrikin's fundamental theorem [2] on finite groups of prime exponent is the following result.

THEOREM 1. Let $L$ be a Lie algebra of characteristic $p$ satisfying the $t$-th Engel condition for some $t<p$, and suppose that $L$ is generated by elements that are right-Engel of length 2. Then $L$ is locally nilpotent.

The main thrust of [2] is the proof of this result without the condition on generation: in that form, it implies the truth of the restricted Burnside problem for exponent $p$. What I want to do here is to present some evidence suggesting that Theorem $1 \mathrm{might}$ be valid without the assumption that $L$ satisfy an Engel condition, but keeping the generation condition. The evidence is not strong enough to state this as a conjecture, but it is enough to state it as a problem.

The fact that $L$ is an algebra over a field seems to be fairly unimportant in our context. What matters is that the additive group $L^{+}$should fail to have involutions. In the first result, we use the abbreviation " $R E_{2}$-element" for "right-Engel element of length 2", so that $x$ is $R E_{2}$ if and only if $[y, x, x]=0$ for all $y$ in $L$.

THEOREM 2. Let $L$ be a Lie ring whose additive group $L^{+}$is 2 -free. If $L$ is generated by 2,3 or $4 R E_{2}$-elements, then $L$ is nilpotent of class at most 2, 3, 5 respectively.

I have been unable to prove such a result even for five generators, but I believe something along these lines will hold for arbitrary generating numbers. The proof of Theorem 2 depends on the following simple Lemma, due essentially to Kostrikin [2].

Lemma 3. Let $L$ be a Lie ring with $L^{+} 2$-free, and let $c$ be an $R E_{2}$-element of $L$. Then $[y, c, u, c]=0$ and $[u, c, x, y, c]=[u, c, y, x, c]$ for all $x, y, u$ in $L$.

Proof. Since $[g, c, c]=0$ for all $g$ in $L$, we have

$$
\begin{aligned}
0=[y,[u, c, c]]= & {[y,[u, c], c]-[y, c,[u, c]] } \\
= & {[y, u, c, c]-[y, c, u, c] } \\
& -[y, c, u, c]+[y, c, c, u] \\
= & -2[y, c, u, c],
\end{aligned}
$$

so that $[y, c, u, c]=0$ since there are no involutions. Using this, we have

$$
0=[u, c,[x, y], c]=[u, c, x, y, c]-[u, c, y, x, c] \text {. }
$$

Now we proceed to the proof of Theorem 2 . Throughout we use the fact that the $n$th

Glasgow Math. J. 21 (1980) 139-142 
term $\gamma_{n}(L)$ of the lower central series of $L$ is the ideal closure of all left-normed products of weight $n$ in the generators of $L$.

For two generators, everything is clear. Now suppose that $L$ is generated by $R E_{2}$-elements $a, b, c$. Possible non-trivial products of weight 4 are, essentially, $[a, b, c, a]$, $[a, b, c, c],[a, b, c, b]$. The last two are zero by definition of $R E_{2}$-elements and the first part of Lemma 3, while

$$
[a, b, c, a]=-[b, a, c, a]=0,
$$

also by Lemma 3.

Suppose finally that we have four $R E_{2}$-generators $a, b, c, d$. By what we have proved so far, there is essentially only one choice for a (possibly!) non-trivial product of weight 4 , viz $[a, b, c, d]$. Again by Lemma 3, a typical product of weight 5 is

$$
[a, b, c, d, b] \text {. }
$$

Adding a further $d$ or $b$ at the end produces the zero element, by Lemma 3. So finally, we have to consider $[a, b, c, d, b, c]$ and $[a, b, c, d, b, a]$. Using both parts of Lemma 3 we get

$$
[a, b, c, d, b, c]=[a, b, d, c, b, c]=0,
$$

and the other product is similarly zero since it is

$$
-[b, c, a, d, b, a]-[c, a, b, d, b, a]=-[b, c, a, b, d, a]=[c, b, a, b, d, a]=0 .
$$

As I mentioned previously, I have been unable to extend this to higher generating numbers. On the other hand, our next result is further evidence, as well as reinforcing the connection between solubility and nilpotency for Lie-type considerations. In fact, very slight variations of the proof will yield that locally soluble Engel Lie rings are locally nilpotent, and also the same result for groups (Gruenberg [1]).

THEOREM 4. Let $L$ be a soluble Lie ring with $L^{+} 2$-free and suppose that $L$ is generated by finitely many $R E_{2}$-elements. Then $L$ is nilpotent.

The proof depends on the following lemmas. We use the notation

$$
\begin{aligned}
& L=\delta_{0}(L) \geqq \delta_{1}(L) \geqq \ldots \geqq \delta_{n}(L) \geqq \ldots \\
& 0=\zeta_{0}(L) \leqq \zeta_{1}(L) \leqq \ldots \leqq \zeta_{n}(L) \leqq \ldots
\end{aligned}
$$

for the derived series and upper central series of a Lie ring $L$.

LEMMA 5. If $L$ is generated by $R E_{2}$-elements and $L^{+}$is 2 -free, then $\delta_{1}(L)^{+}$is generated by $R E_{2}$-elements.

Proof. All we need to do is to prove that the product of two $R E_{2}$-elements is an $R E_{2}$-element. This follows easily from Lemma 3; for any $R E_{2}$-elements $x, y$ and any 
element $u$,

$$
\begin{aligned}
{[u,[x, y],[x, y]]=} & {[u, x, y,[x, y]]-[u, y, x,[x, y]] } \\
= & {[u, x, y, x, y]-[u, x, y, y, x] } \\
& -[u, y, x, x, y]+[u, y, x, y, x]=0 .
\end{aligned}
$$

The next result is well-known and its analogue in group theory much used; we omit the proof.

Lemma 6. Let $L$ be a Lie ring, $H$ an ideal annihilating $\delta_{1}(L)$. Then for all $h \in H$, all $x_{1}, \ldots, x_{n}$ in $L$ and permutations $\pi$ of $\{1,2, \ldots, n\}$,

$$
\left[h, x_{1}, x_{2}, \ldots, x_{n}\right]=\left[h, x_{1 \pi}, x_{2 \pi}, \ldots, x_{n \pi}\right] \text {. }
$$

We can now prove Theorem 4 by induction on the solubility length of $L$. Suppose first that $L$ is metabelian, and let $g_{1}, \ldots, g_{k}$ be a generating set for $L$ consisting of $R E_{2}$-elements. Then $\left[x, g_{i}, g_{i}\right]=0$ for all $x$ in $L$, and Lemma 6 with $H=\delta_{1}(L)$ gives that $\left[h, g_{i_{1}}, g_{i_{2}}, \ldots, g_{i_{k+1}}\right]=0$ for any $i_{1}, i_{2}, \ldots, i_{k+1}$ and each $h$ in $H$, since some $g_{i}$ must be repeated. Thus every element of $\delta_{1}(L)$ is in the $(k+1)$-st term of the upper central series of $L$, so that $L$ is nilpotent of class at most $k+2$. (In fact, this part of the proof works when the generating elements are right Engel of any length).

Now suppose that $L$ is of solubility length $n \geqq 2$ and that Theorem 4 has been proved for lower solubility lengths. We shall first show that $\delta_{1}(L)$ is nilpotent.

By induction, $L / \delta_{n-1}(L)$ is nilpotent, so that $\gamma_{c}(L) \leqq \delta_{n-1}(L)$ for some $c$, and $\delta_{1}(L) / \delta_{n-1}(L)$ is finitely generated. Since $\delta_{1}(L)$ is generated by $R E_{2}$-elements, there is a finite generating set $x_{1}, x_{2}, \ldots, x_{1}$ of $\delta_{1}(L)$ modulo $\delta_{n-1}(L)$ consisting of $R E_{2}$-elements. Now $\gamma_{c}(L)$ is the ideal closure of finitely many elements $h_{1}, h_{2}, \ldots, h_{d}$, each of which is an $R E_{2}$-element (namely, a product of weight $c$ in the original generators of $L$ ). Thus by induction the elements

$$
x_{1}, x_{2}, \ldots, x_{l}, h_{1}, \ldots, h_{d}
$$

of $\delta_{1}(L)$ generate a nilpotent subring, of class $e$, say. Hence every product

$$
\left[h_{1}, f_{1}, f_{2}, \ldots, f_{e}\right] \text {, }
$$

with $f_{i}$ either an $x_{i}$ or an element of $\delta_{n-1}(L)$, is trivial. Thus $h_{i} \in \zeta_{e}\left(\delta_{1}(L)\right)$, so that

$$
\zeta_{e}\left(\delta_{1}(L)\right) \geqq \gamma_{c}(L) \geqq \gamma_{c}\left(\delta_{1}(L)\right),
$$

which means that $\delta_{1}(L)$ is nilpotent.

Next, put $K=L /\left[\delta_{n-1}(L), \delta_{1}(L)\right]$. Then $\delta_{n-1}(K)$ is an abelian ideal annihilating $\delta_{1}(K)$, so that every element of $\delta_{n-1}(K)$ is in some term of the upper central series of $K$. But $\gamma_{c}(K) \leqq \delta_{n-1}(K)$ and $\gamma_{c}(K)$ is the ideal closure of finitely many elements, so again $\gamma_{c}(K)$ is in some term of the upper central series of $K$. But $\gamma_{c}(K) \leqq \delta_{n-1}(K)$ and $\gamma_{c}(K)$ is the ideal closure of finitely many elements, so again $\gamma_{c}(K)$ is in some term of the upper central series of $K$. Thus $K$ is nilpotent, and $\left[\delta_{n-1}(L), \delta_{1}(L)\right]$ contains some term of the lower central series of $L$. Continuing in this way, we can prove that $\left[\delta_{n-1}(L), \delta_{1}(L), \ldots, \delta_{1}(L)\right]$, 
with any number of repeats of $\delta_{1}(L)$, contains some term of the lower central series of $L$. Since $\delta_{1}(L)$ is nilpotent, this means that $L$ is nilpotent, and the induction is complete.

\section{REFERENCES}

1. K. W. Gruenberg, Two theorems on Engel groups, Proc. Cambridge Philos. Soc. 49 (1953), 377-380.

2. A. I. Kostrikin, On Burnside's Problem, Izv. Akad. Nauk. SSSR. Ser. Mat. 23 (1959), 3-34.

UnIVERSITY COLLEGE,

CARdifF,

WALES 